\title{
Staging of uterine cervical cancer with MRI: guidelines of the European Society of Urogenital Radiology
}

\author{
Corinne Balleyguier - E. Sala • T. Da Cunha • A. Bergman • B. Brkljacic • F. Danza • \\ R. Forstner • B. Hamm • R. Kubik-Huch • C. Lopez • R. Manfredi • J. McHugo • \\ L. Oleaga $\cdot$ K. Togashi $\cdot$ K. Kinkel
}

Received: 27 July 2010 /Revised: 29 September 2010 / Accepted: 1 October 2010 /Published online: 10 November 2010

(C) European Society of Radiology 2010

\begin{abstract}
Objective: To design clear guidelines for the staging and follow-up of patients with uterine cervical cancer, and to provide the radiologist with a framework for use in multidisciplinary conferences. Methods: Guidelines for uterine cervical cancer staging and follow-up were defined by the female imaging subcommittee of the ESUR
\end{abstract}

\section{Balleyguier $(\bowtie)$}

Radiology Department, Institut Gustave Roussy,

39, rue Camille Desmoulins,

94805 Villejuif, Cedex, France

e-mail: corinne.balleyguier@igr.fr

\section{E. Sala}

Radiology Department, Addenbrooke's Hospital,

Cambridge, UK

\section{T. Da Cunha}

Radiology Department, Instituto Português de Oncologia de

Lisboa Francisco Gentil,

R. Prof. Lima Basto,

Lisbon, Portugal

\section{A. Bergman}

Department of Radiology, Uppsala University Hospital,

Uppsala, Sweden

B. Brkljacic

Department of Diagnostic and Interventional Radiology,

University Hospital "Dubrava",

Zagreb, Croatia

\section{F. Danza}

Dipartimento di Bioimmaginie scienze radiologiche, Università

Cattolica del S. Cuore,

Rome, Italy

\section{R. Forstner}

Zentralröntgeninstitut, LandesklinikenSalzburg,

Salzburg, Austria
(European Society of Urogenital Radiology) based on the expert consensus of imaging protocols of 11 leading institutions and a critical review of the literature. Results: The results indicated that high field Magnetic Resonance Imaging (MRI) should include at least two T2-weighted sequences in sagittal, axial oblique or coronal oblique

\section{B. Hamm}

Department of Radiology, Charité Humboldt Universität Berlin, Berlin, Germany

R. Kubik-Huch

Institut Radiologie Kantonsspital Baden,

Baden, Germany

C. Lopez $\cdot$ R. Manfredi

Department of Radiology, “A. Gemelli” University Hospital, Rome, Italy

J. McHugo

Department of Radiology, Birmingham Women's Hospital, Birmingham, UK

L. Oleaga

Radiology Department, Hospital Clinic,

C/Villaroel 170,

Barcelona, Spain

K. Togashi

Department of Diagnostic Imaging and Nuclear Medicine, Kyoto University Graduate School of Medicine,

Kyoto, Japan

K. Kinkel

Institut de radiologie, Clinique des Grangettes,

Chêne-Bougerie/Geneva, Switzerland 
orientation (short and long axis of the uterine cervix) of the pelvic content. Axial T1-weighted sequence is useful to detect suspicious pelvic and abdominal lymph nodes, and images from symphysis to the left renal vein are required. The intravenous administration of Gadolinium-chelates is optional but is often required for small lesions $(<2 \mathrm{~cm})$ and for follow-up after treatment. Diffusion-weighted sequences are optional but are recommended to help evaluate lymph nodes and to detect a residual lesion after chemoradiotherapy. Conclusions: Expert consensus and literature review lead to an optimized MRI protocol to stage uterine cervical cancer. MRI is the imaging modality of choice for preoperative staging and follow-up in patients with uterine cervical cancer.

Keywords Uterus · Cervix · Cervical cancer · Staging · Magnetic resonance (MR)

\section{Introduction}

Uterine cervical cancer is the third most common gynaecological malignancy after endometrial and ovarian cancers [1]. Nearly $80 \%$ of cervical cancer occurs in the developing countries and most patients are diagnosed with the disease at an advanced stage, thus not suitable for surgical staging. Therefore cervical cancer usually remains a clinically staged disease [2]. However, there are significant inaccuracies in the clinical staging system compared to surgical staging, with an error rate up to $32 \%$ in patients with stage IB disease and up to $65 \%$ of patients with stage III disease [3]. The greatest difficulties in the clinical examination of patients with cervical cancer are accurate estimation of tumour size, the assessment of parametrial and pelvic side wall invasion, and the evaluation of lymph node metastases which are recognized important prognostic factors [4]. Despite these inherent limitations of the clinical examination, uterine cervical cancer is the only gynaecological cancer still largely staged clinically, in accordance with the classification of the International Federation of Gynaecology and Obstetrics (FIGO).

In June 2009 the FIGO committee introduced the revised staging [5] of cervical carcinoma updating the previous staging of 1988 (Tables 1 and 2). Although the revised FIGO staging system does not include imaging in the staging of cervical cancer, for the first time the committee encourages the use of imaging techniques, if available, to assess the important prognostic factors such as tumour size, parametrial and pelvic side wall invasion, adjacent organ invasion and the evaluation of lymph node metastases. Imaging is thus complementary to the clinical assessment with MRI being the optimal modality to stage cervical carcinoma FIGO stage 1B1 or greater. Furthermore, in young patients with small invasive tumours and who desire to preserve fertility a more conservative surgical procedure (trachelectomy) can be performed. In these cases MRI staging is mandatory in order to determine eligibility in terms of tumour size $(<2 \mathrm{~cm})$, cervical length $(>2.5 \mathrm{~cm})$ and distance from the tumour to

Table 1 New FIGO classification for cervical carcinoma. FIGO COMMITTEE ON GYNECOLOGIC ONCOLOGY International Journal of Gynecology and Obstetrics 105 (2009) 103-104

Stage I: The carcinoma is strictly confined to the cervix (extension to the corpus would be disregarded)

IA Invasive carcinoma which can be diagnosed only by microscopy, with deepest invasion $\leq 5 \mathrm{~mm}$ and largest extension $\geq 7 \mathrm{~mm}$

IA1 Measured stromal invasion of $\leq 3.0 \mathrm{~mm}$ in depth and extension of $\leq 7.0 \mathrm{~mm}$

IA2 Measured stromal invasion of $\mathrm{N} 3.0 \mathrm{~mm}$ and not $\mathrm{N} 5.0 \mathrm{~mm}$ with an extension of not $\mathrm{N} 7.0 \mathrm{~mm}$

IB Clinically visible lesions limited to the cervix uteri or pre-clinical cancers greater than stage IA *

IB1 Clinically visible lesion $\leq 4.0 \mathrm{~cm}$ in greatest dimension

IB2 Clinically visible lesion $\mathrm{N} 4.0 \mathrm{~cm}$ in greatest dimension

Stage II: Cervical carcinoma invades beyond the uterus, but not to the pelvic wall or to the lower third of the vagina

IIA Without parametrial invasion

IIA1 Clinically visible lesion $\leq 4.0 \mathrm{~cm}$ in greatest dimension

IIA2 Clinically visible lesion N4 cm in greatest dimension

IIB With obvious parametrial invasion

Stage III: The tumour extends to the pelvic wall and/or involves lower third of the vagina and/or causes hydronephrosis or non-functioning kidney IIIA Tumour involves lower third of the vagina, with no extension to the pelvic wall

IIIB Extension to the pelvic wall and/or hydronephrosis or non-functioning kidney

Stage IV: The carcinoma has extended beyond the true pelvis or has involved (biopsy proven) the mucosa of the bladder or rectum. A bullous edema, as such, does not permit a case to be allotted to Stage IV

IVA Spread of the growth to adjacent organs

IVB Spread to distant organs 
Table 2 Proposed protocol for MRI in cervical cancer staging and evaluation

- Optional use of fasting, Antiperistaltic IV injection, or vaginal/rectal sonographic gel opacification

- Axial T2w sequence, without a fat suppression : pelvis and abdomen (renal hilum) : $5 \mathrm{~mm} / 0.5$ (pelvis), $6 \mathrm{~mm} / 1 \mathrm{~mm}$ (abdomen) ; matrix $>$ $400 \times 400$

- Sagittal T2w sequence without a fat suppression : pelvis $5 \mathrm{~mm} / 0.5$ (pelvis) ; matrix $>400 \times 400$

- Coronal oblique $\mathrm{T} 2 \mathrm{w}$ perpendicular to the cervix, thin slices $(4 \mathrm{~mm} / 0.4 \mathrm{~mm})$; matrix $>400 \times 400$

- Optional: in case of small lesions, not well depicted on T2w images, and after treatment.

- Sagittal 3D T1w dynamic sequences after gadolinium chelates injection : one native and 4 post contrast (total 5 scans with a total acquisition time of about $5 \mathrm{mn}$ )

- DWI sequences

the internal cervical os $(>1 \mathrm{~cm})$. In order to achieve maximal staging accuracy by means of MRI, it is essential to ensure adequate patient preparation, optimal imaging sequences and adequate reporting expertise. Therefore, the female pelvis subcommittee of the European Society of Urogenital Radiology (ESUR) formed a working group to establish technical guidelines for uterine cervical cancer staging with MRI based on extended clinical practice.

\section{Material and methods}

MRI protocols for staging of cervical cancer were collected from eleven European Institutions. Inclusion criteria to participate in the guideline process were: to be a member of the European Society of Urogenital Radiology (ESUR) and to perform at least ten MRI examinations per year for staging biopsy-proven cervical carcinoma. Members of the ESUR are experts in gynaecological imaging, especially in pelvic MRI. The questionnaire contained the following information: patient preparation, magnet field strength, type of coil, type of sequence with detailed geometry and contrast information, such as FOV, matrix scan and reconstruction, slice thickness, gap, orientation, saturation bands, 2D versus 3D sequence, TR/ $\mathrm{TE}$, use of number of acquisitions, number and lengths of dynamic sequences, use of bolus intravenous (IV) injection and subtraction techniques and use of diffusion weighted imaging (DWI) sequences. In addition, published literature between 1999 and 2010 was reviewed through a Medline literature search of abstracts in the English language of studies in human subjects, including the following key words: "Uterine neoplasm(s) AND MR imaging" and "Uterine cervical carcinoma AND MR imaging." Articles that did not include technical details matching the information requested in the questionnaire were excluded. The details were entered in an excel sheet and the results were discussed and divided in topics with agreement and disagreement. Topics with disagreement were compared to the literature. Experts in favour of one technical option were asked to support their views using data from the literature. These were subsequently discussed by an expert panel. The collected data from the ESUR member institution was compared to the data from the literature which took into consideration year of publication, number of cases and performance of the technique. When technical details varied and no published work on performance was found, the subject was discussed and resolved in consensus based on the majority of participating members. Members of the group met twice a year during a 2-year period and were asked to apply technical recommendations issued after the first two meetings during the year before the meetings in year 2 in order to allow further discussion and the issue of final recommendations in consensus. Validation of the manuscript has been done collegially with the agreement of the ESUR committee.

\section{Results}

Indications for MRI in uterine cervical cancer

All the eleven ESUR members recommend MRI for staging of tumours stage 1B1 and over or smaller tumours if trachelectomy is being considered. They also all recommended MRI for treatment follow-up (after brachytherapy and chemoradiation therapy) and detection of tumour recurrence. There was no consensus among 11 ESUR participants regarding the MRI time-delay after treatment. This varied from 3 weeks to 6 months with the majority recommending MRI 3-6 months after completion of chemoradiation therapy and brachytherapy. Similarly, there was no consensus on the frequency of follow-up. Some ESUR members perform yearly follow-up MRI for 5 years and some only if there are symptoms of recurrence. Three members use PET/CT routinely as complementary to MRI staging, 5 only if there are indeterminate lymph nodes on MRI and 2 only before salvage therapy.

Technical requirements for MRI in uterine cervical cancer

Among the eleven ESUR members, 6 worked with a $1.5 \mathrm{~T}$ magnet, 1 with a 3.0 T magnet and 4 members with both 1.5 and 3.0 $\mathrm{T}$ magnet, Most of the published studies in the literature use a $1.5 \mathrm{~T}$ magnet. There are only a few published 
studies using a $3 \mathrm{~T}$ MRI, showing very promising results in cervical cancer [6]. At $3 \mathrm{~T}$, spatial resolution is improved, allowing accurate local staging and detection of a residual tumour after treatment [6]. At 3.0-T imaging, mean tumour signal-to-noise ratios, mean cervical stroma signal-to-noise ratios, and mean tumour-to-cervical stroma contrast-to-noise ratios are higher than those at $1.5-\mathrm{T}$ imaging. Nevertheless, image homogeneity at 3.0-T imaging seems inferior to that at 1.5-T imaging. In recent published studies, even if imaging quality seems to be higher at $3 \mathrm{~T}$, accuracy seems to be equivalent at $1.5 \mathrm{~T}$ and $3 \mathrm{~T}(6)$.

\section{a. Patient Preparation}

There was no consensus among the 11 ESUR members or in the literature regarding the type of preparation before pelvic MRI. Nevertheless, different types of patient preparations have been suggested in order to improve the quality of the examination. Several authors proposed a $6 \mathrm{~h}$ fast before MRI $[7,8]$ others an IV or intramuscular (IM) injection of antiperistaltic agent [9] or vaginal/rectal opacification with sterile gel [10]. The use of an antiperistaltic agent seems to be the most efficient way to limit bowel motion artifacts. An intramuscular injection of an antiperistaltic agent is used (1 mg glucagon; (Glucagen ${ }^{\circledR}$, Novo Nordisk ${ }^{\circledR}$; Bagsværd ; Denmark) or $20 \mathrm{mg}$ butyl-scopolamine (Buscopan ${ }^{\circledR}$; Boehringer Ingelheim $\mathrm{GmbH}$; Ingelheim, Germany) unless contraindicated (e.g., diabetes or pheochromocytoma) to decrease peristalsis artifacts. In the ESUR group, only $50 \%$ of the experts included combined fasting and antiperistaltic agents in their patient preparation protocol. Seven ESUR centres use an antiperistaltic agent routinely before MRI examination (4 use IV injection and 3 use IM injection) whereas four centres do not use an antiperistaltic agent. Bladder must be half filled in order to improve lesion visibility without changing anatomy. Vaginal opacification with gel yielding high signal intensity on T2 weighting may be useful in case of suspicion of tumour extension into the vagina in order to differentiate FIGO IIA from FIGO IB tumour, particularly in the posterior vaginal fornix [10]; however only one ESUR centre uses a vaginal opacification routinely and two others only if there is a suspicion of posterior fornix invasion at physical examination. Therefore, the use of intraluminal (vagina/rectum) contrast agents before the MRI staging of uterine cervical carcinoma remains optional.

\section{b. Use of Intravenous Contrast Medium}

There is wide variation among the 11 ESUR members and in the literature regarding the use of IV contrast medium for cervical cancer staging. Seven of the 11 ESUR members use IV contrast medium routinely for staging of cervical cancer and identification of small tumours, one uses it only for detection of tumour recurrence and three do not use IV) contrast medium. Among those 7 centres that use IV contrast medium, 6 use a dynamic acquisition and 1 uses a semidynamic one. The results of the literature are also variable with some authors using IV contrast medium systematically in all cases [10-12] whereas other authors not using IV contrast medium at all $[13,14]$. In a dynamic acquisition, contrast enhancement of the tumour is lower than the myometrium in the early phase, whereas on the late phases, tumour signal intensity is higher than the myometrium. Thus, interpretation of images is easier on the earlier phases of contrast medium enhancement. The use of IV contrast medium increases the contrast between the tumour and normal cervical stroma and can improve tumour detection and localization, this is especially useful for small tumours which may be considered for trachelectomy [15]. Van Vierzen et al [15] found that the combination of pre-contrast and post-contrast MR images did not clearly improve staging accuracy (83\%). However, the addition of fast dynamic contrast-enhanced MRI improved staging accuracy to $91 \%$. The use of dynamic contrast-enhanced MRI also improves the accuracy of assessment of bladder and rectal wall invasion [15]. Furthermore, the use of IV contrast medium is useful in the post-treatment setting to differentiate residual or recurrent tumour from radiation fibrosis [16] as well as presence of fistulae occurring post radiation therapy.

\section{c. Imaging Sequences}

There is significant variability in the literature regarding the MRI protocols used. The number of the sequences can vary from 3 [17] to more than 5 [18]. There was a consensus in the ESUR group that the essential protocol must include a combination of at least two T2-weighted sequences obtained in the sagittal and oblique (perpendicular to the cervical canal) planes and T1W sequences of the upper abdomen and pelvis. Lymph node evaluation might be done also on T2 sequences in an axial plan, from pelvis to left renal vein.

T2w sequences are the best sequences to detect the cervical tumour and its extension to the uterus, parametria and the adjacent organs [19] (Fig. 1). The use of fat suppression is not recommended as the presence of parametrial fat can lead to better tumour delineation. All the ESUR centres and most of the authors in the literature are performing $\mathrm{T} 2 \mathrm{w}$ sequences in two orthogonal planes such as sagittal and oblique [19]. Oblique images must be acquired perpendicularly to the long axis of the cervical canal. Slice thickness varied from 3 to $6 \mathrm{~mm}$, with a 0.25 gap. For optimal image quality, T2-weighted images covering the pelvis should be acquired with a small FOV $(20-25 \mathrm{~cm})$ and ideally with a $512 \times 512$ matrix. 8 of 18 reviewed papers also add a coronal $\mathrm{T} 2 \mathrm{w}$ sequence on the pelvis with thin slices $(3-4 \mathrm{~mm} / 0.4 \mathrm{~mm})$ to assess parametrial extension [20] (Fig. 2). All the ESUR centres and several authors in the literature are also obtaining images of 


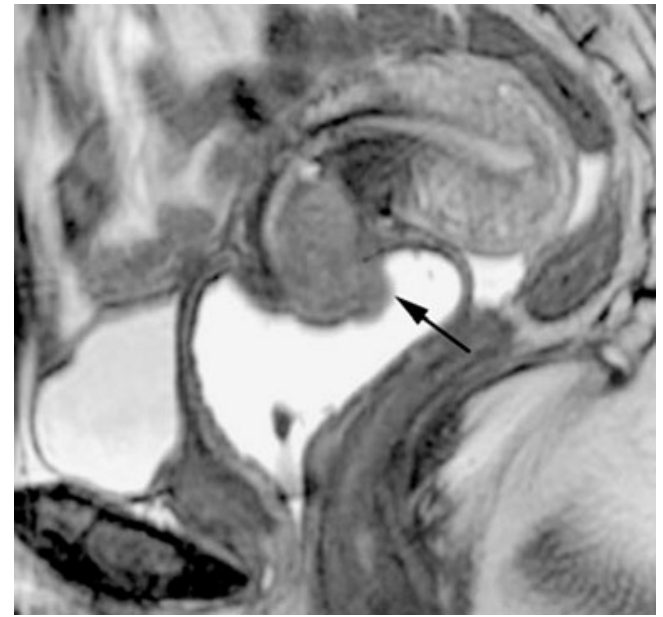

Fig. 1 Sagittal T2w image, MRI. 45 years old woman, stage IB2 cervical carcinoma. T2W images demonstrate the tumour (arrow) invading the cervical stroma. Vaginal opacification with gel yielding high signal intensity on $\mathrm{T} 2$ weighting does not depict any vaginal invasion

the abdomen (from the level of the renal veins to the pelvic brim) to evaluate for presence of abnormal lymph nodes [7, 19, 21] (Fig. 3). Both T1 and T2 - weighted images can be used for this purpose. This obviates the need for an additional $\mathrm{CT}$ of the abdomen. The total acquisition time for the entire staging MRI is $25-30 \mathrm{~min}$.

T1w sequences were part of the MRI staging protocol with 14/18 reviewed papers and nearly all members of the ESUR group performing T1w images without fat suppression. T1w sequences are very useful to evaluate for presence of lymphadenopathy, bone metastases and also in the rare occasion of hematometria. Therefore, they should be mandatory. Nevertheless, lymph node evaluation can also be done accurately on $\mathrm{T} 2 \mathrm{w}$ axial sequences.

Diffusion weighted imaging (DWI) Although there is little published data, DWI seems to be a very promising

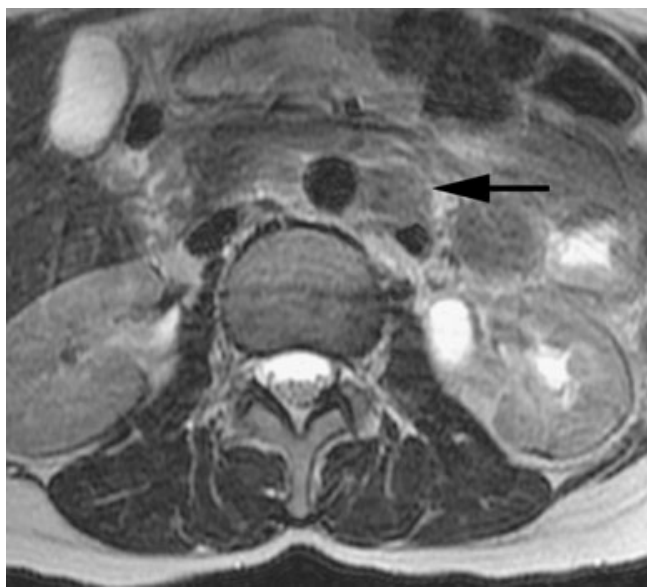

Fig. 3 Axial T2W image, MRI. 47 years old woman, stage IIB cervical carcinoma. Lymph node spreading. An abnormal lymph node (small axis> $10 \mathrm{~mm}$ ) is seen in the para-aortic space (arrow). It is mandatory to perform in the same examination a pelvic and an abdominal staging, with slices from the symphysis to the left renal vein

emerging technique in the evaluation of uterine cervical cancer [8, 22-25]. Seven ESUR centres routinely use DWI. Cervical carcinoma has a lower apparent diffusion coefficient (ADC) compared to the normal cervix (Fig. 4). The ADC increases after chemoradiotherapy [26]. There is a variability in the B-values used with a range of $\mathrm{B}$ values between 500 and $1000 \mathrm{bms} /-2$ [26, 27]. DWI may be helpful in detection of residual tumour or suspicious lymph nodes after chemoradiotherapy [8], and might be competitive with PET-imaging $[28,29]$. A recent paper showed that the pretreatment ADCs of patients with complete response are lower than that of those with partial response, and pretreatment $\mathrm{ADC}$ values of all patients correlated negatively with the percentage size reduction of the tumour after 2 months of chemoradiation. A possible explanation for this observation is that tumours with high pretreatment ADC values are likely to be more necrotic than those with low
Fig. 2 Sagittal and axial T2W images, MRI. 38 years old woman, stage IB2 cervical carcinoma. Hyperintense lesion is invading the cervical stroma (Fig 2a). Coronal oblique section shows an intact low signal intensity cervical stroma (arrows, Fig 2b) accurately excluding presence of parametrial invasion
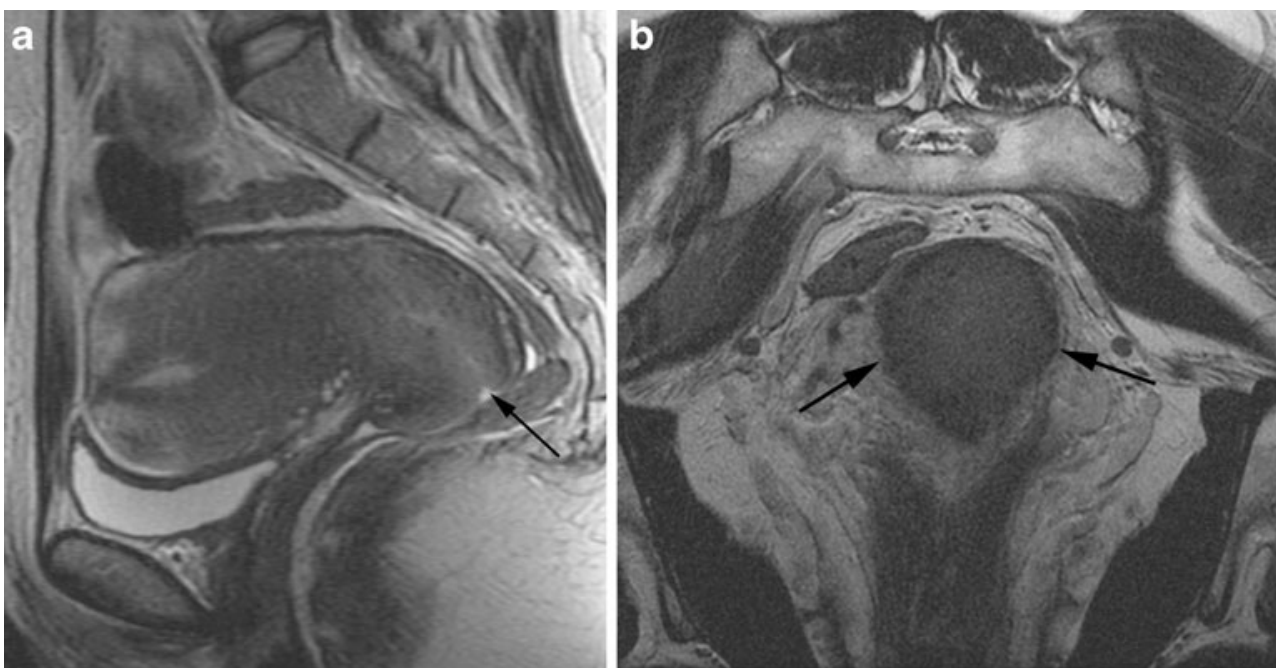

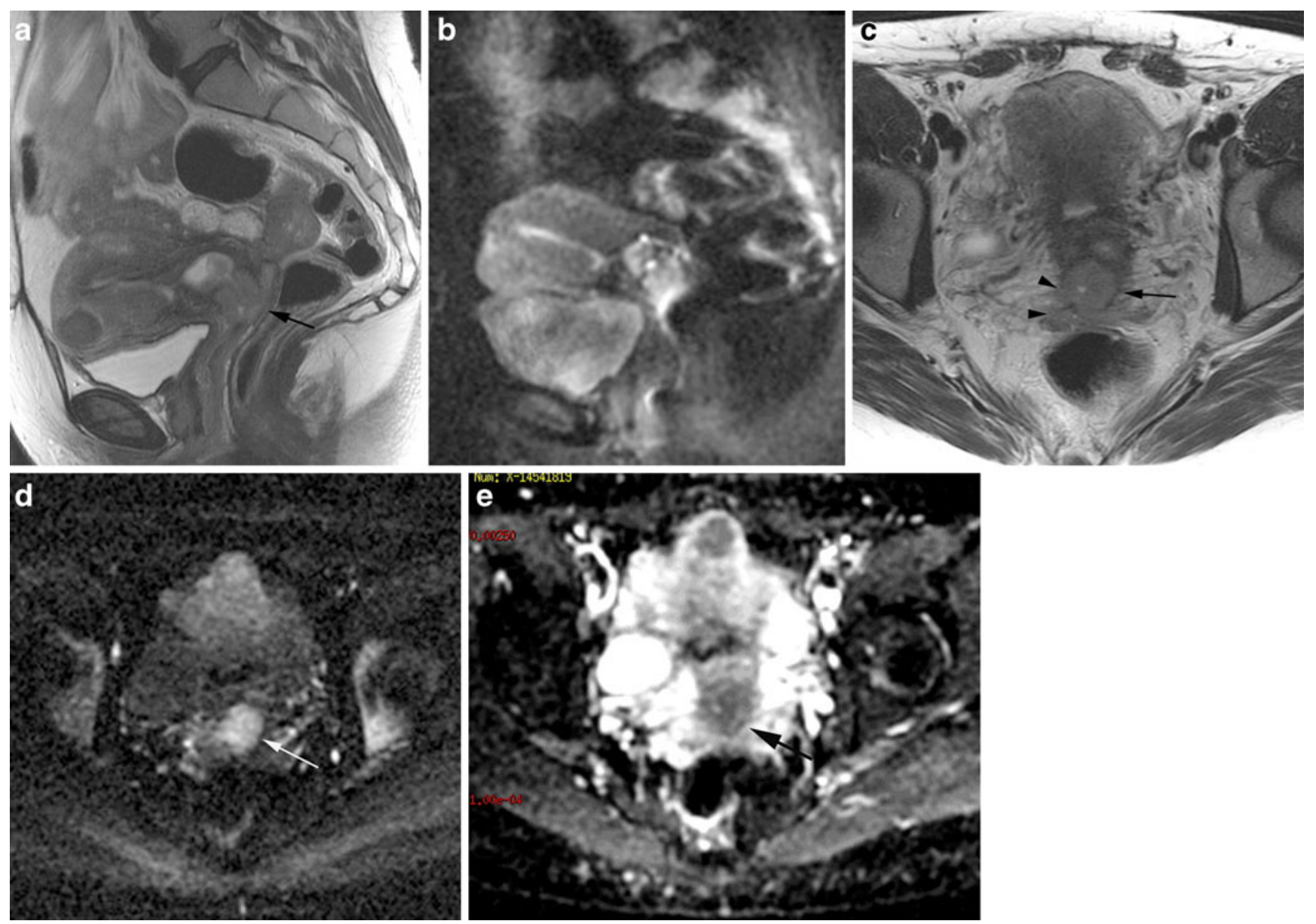

Fig. 4 Sagittal and axial T2W images, MRI; DWI images with ADC map (B 800). 42 years old woman, stage IIB cervical carcinoma. A $2 \mathrm{~cm}$ hyperintense lesion is seen in the cervix (arrow) (Fig 4a) with right parametrial invasion (Fig 4c) (arrowheads) and a beginning of left parametrial invasion (arrow). Sagittal and axial DWI images (Fig $4 \mathbf{b}$ ) and Fig 4d show high signal intensity on DWI and with a low signal intensity on the ADC map (Fig 4e) suggesting restricted diffusion

b. Local staging

ADC values [8]. We recommend performing optional DWI sequences of the pelvis and the abdomen.

\section{Reporting}

The following check list is helpful for a comprehensive and easy to read report.

\section{a. Description of the lesions}

Cervical tumour is depicted on $\mathrm{T} 2 \mathrm{w}$ images as a hyperintense mass compared to the cervical stroma [30, 31]. Tumour size (in three dimensions) must be evaluated in at least two orthogonal views; it is crucial to give precise measurements as size of the tumour can dictate treatment options. Fertility sparing surgery is possible with tumours $<2 \mathrm{~cm}$ whereas patients with tumours $>4 \mathrm{~cm}$ may undergo chemoradiotherapy rather than radical surgery.
- Vaginal extension: precise description should be given if the extension is anterior or posterior. If the lesion reaches the $2 / 3$ of the upper vagina, it is a FIGO IIA; if the lesion invades the $1 / 3$ inferior part of the vagina, it is a FIGO IIIA.

- Parametrial extension: stromal invasion is present if there is a disruption of the hypointense line circumscribing the cervix on oblique T2w images (Fig. 5). Parametrial invasion is present if in addition to the stromal invasion there is tumour present in the parametrium, a spiculated tumourparametrial interface or tumour encasement of the peri-uterine vessels [12]. Presence of hydronephrosis is consistent with parametrial invasion. Accuracy of MRI in assessing parametrial invasion varies from 80 to $87 \%$ according to the literature [32]. 


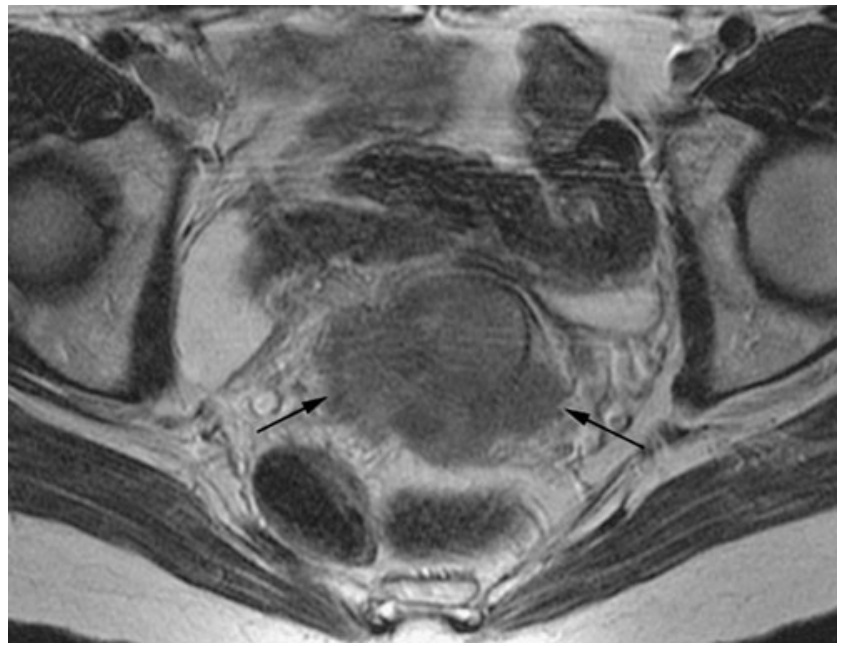

Fig. 5 Axial T2W image, MRI. 49 years old woman, stage IIB cervical carcinoma. Parametrial tumoural extension is seen on both sides of uterine cervix (arrows). Limits of cervical stroma are nearly not visible

- Isthmic extension: clinical evaluation is not accurate in case of isthmic invasion. The reporting should include it because positioning of brachytherapy coils might be dependant of the level of isthmic tumour extension [33].

\section{c. Lymph node staging}

FIGO classification does not include the nodal status. Yet, lymph node spreading is one of the most important prognostic factors in cervical carcinoma [34, 35]. Accuracy of MRI is low for lymph node staging, with a sensitivity varying from 38 to $89 \%$, and a specificity from 78 to $99 \%$ $[36,37]$. Due to its high sensitivity, PET-CT is recommended for locally advanced cervical carcinoma with no abnormal lymph node depicted in the pelvis or the abdomen [38]. Size criterion for a suspicious lymph node is a short axis superior to $1 \mathrm{~cm}$, in the pelvis or the abdomen [39].
However, smaller lymph nodes may be malignant, especially in the pelvis; therefore it is important to take in account other features of malignancy such as round shape, irregular margins, signal intensity similar to the primary tumour and presence of necrosis.

\section{d. Evaluation of Tumour Response to Treatment}

Chemoradiotherapy followed by brachytherapy is the standard treatment for patients with locally advanced uterine cervical carcinoma ( $>$ IB1 FIGO stage) [40]. Surgery is usually avoided in case of complete response following chemoradiotherapy treatment, due to its high rate of urinary side effects (incontinence, uteral distension, chronic bladder infection ...). Tumour response evaluation is based on three criteria: clinical examination, PAP smear analysis and post treatment MR evaluation [41].

After treatment, MR protocol is the same as for cervical cancer staging, but IV injection is recommended. MR criteria for a complete response include:

- no lesion seen in the cervix or in the adjacent anatomic areas

- homogeneous hypointense cervical stroma

- homogeneous and delayed intravenous contrast medium uptake of the cervix after IV injection [42] (Fig. 6).

Interpretation and performance of MRI in the follow-up setting are lower compared to the primary staging tumour detection. It is useful to compare the post treatment images with the pre-treatment images to facilitate tumour detection and re-staging.

e. Detection of a recurrence

- After medical treatment:

There is no consensus among the ESUR centres on in the reviewed literature regarding the indication of MRI for the follow-up of patients with treated cervical carcinoma.
Fig. 6 Sagittal T2W image before $\mathbf{a}$ and after $\mathbf{b}$ radiation-chemotherapy (RCT) and brachytherapy. 37 years old woman, stage IIB cervical carcinoma. A $5 \mathrm{~cm}$ lesion invading the cervical stroma is seen with a hyperintense signal intensity (arrow, fig a). After treatment ( 3 weeks delay), the tumour is not seen. There is reconstitution of the normal low signal intensity cervical stroma suggestive of complete response to treatment. No residual tumour was found at hysterectomy
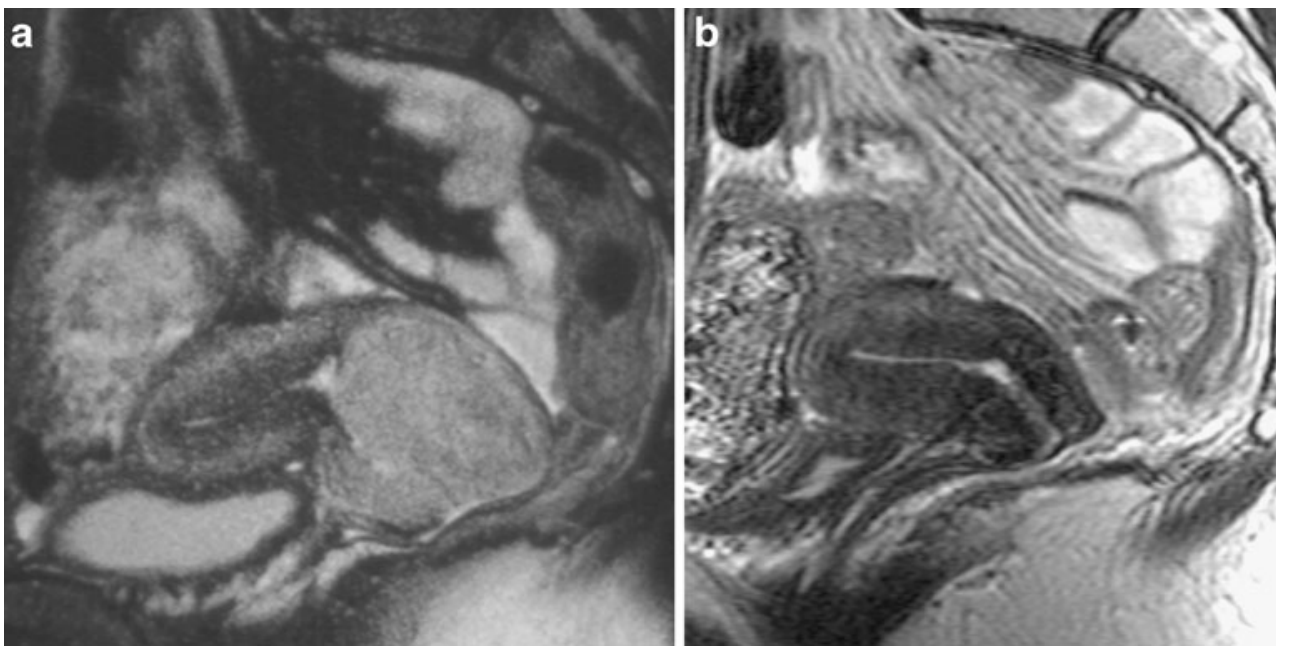
Usually, MRI is performed when there is a clinical suspicion of recurrence.

\section{- After surgery :}

There is also no consensus for a systematic MR followup. Nevertheless, in case of trachelectomy, an MRI at 6 months and 1 year is advised, because recurrence rate is higher compare to patients who undergo brachytherapy or chemotherapy [43].

\section{Conclusion}

Although FIGO staging system does not include imaging in the staging of cervical cancer, in the revised FIGO system imaging techniques are encouraged to assess the important prognostic factors and imaging is now complimentary to the clinical assessment. Radiologists must familiarize themselves with the new FIGO staging system and understand its relevance to patient management. Magnetic resonance imaging is the imaging modality of choice for staging the primary cervical tumour, evaluate response to treatment and detect tumour recurrence and potential complications. Adequate patient preparation, protocol optimization and MRI reporting expertise are essential to achieve high diagnostic accuracy.

\section{References}

1. Ozsarlak O, Tjalma W, Schepens E, Corthouts B, Op de Beeck B, Van Marck E et al (2003) The correlation of preoperative CT, MR imaging, and clinical staging (FIGO) with histopathology findings in primary cervical carcinoma. Eur Radiol 13:2338-2345

2. Odicino F, Tisi G, Rampinelli F, Miscioscia R, Sartori E, Pecorelli S (2007) New development of the FIGO staging system. Gynecol Oncol 107(1 Suppl 1):S8-S9

3. Lagasse LD, Creasman WT, Shingleton HM, Ford JH, Blessing JA (1980) Results and complications of operative staging in cervical cancer: experience of the Gynecologic Oncology Group. Gynecol Oncol 9:90-98

4. Piver MS, Chung WS (1975) Prognostic significance of cervical lesion size and pelvic node metastases in cervical carcinoma. Obstet Gynecol 46:507-510

5. Pecorelli S (2009) Revised FIGO staging for carcinoma of the vulva, cervix, and endometrium. Int J Gynaecol Obstet 105:103-104

6. Hori M, Kim T, Murakami T, Imaoka I, Onishi H, Tomoda K et al (2009) Uterine cervical carcinoma: preoperative staging with 3.0T MR imaging-comparison with 1.5-T MR imaging. Radiology 251:96-104

7. Engin G, Cervical cancer (2006) MR imaging findings before, during, and after radiation therapy. Eur Radiol 16:313-324

8. Liu Y, Bai R, Sun H, Liu H, Zhao X, Li Y (2009) Diffusionweighted imaging in predicting and monitoring the response of uterine cervical cancer to combined chemoradiation. Clin Radiol 64:1067-1074

9. Haider MA, Patlas M, Jhaveri K, Chapman W, Fyles A, Rosen B (2006) Adenocarcinoma involving the uterine cervix: magnetic resonance imaging findings in tumours of endometrial, compared with cervical, origin. Can Assoc Radiol J 57:43-48

10. Van Hoe L, Vanbeckevoort D, Oyen R, Itzlinger U, Vergote I (1999) Cervical carcinoma: optimized local staging with intravaginal contrast-enhanced MR imaging-preliminary results. Radiology 213:608-611

11. Haider MA, Sitartchouk I, Roberts TP, Fyles A, Hashmi AT, Milosevic M (2007) Correlations between dynamic contrastenhanced magnetic resonance imaging-derived measures of tumour microvasculature and interstitial fluid pressure in patients with cervical cancer. J Magn Reson Imaging 25:153-159

12. Hricak H, Yu KK, Powell CB, Subak LL, Stem J, Arenson RL (1996) Comparison of diagnostic studies in the pretreatment evaluation of stage Ib carcinoma of the cervix. Acad Radiol 3 (Suppl 1):S44-S46

13. deSouza NM, Dina R, McIndoe GA, Soutter WP (2006) Cervical cancer: value of an endovaginal coil magnetic resonance imaging technique in detecting small volume disease and assessing parametrial extension. Gynecol Oncol 102:80-85

14. Rockall AG, Ghosh S, Alexander-Sefre F, Babar S, Younis MT, Naz S et al (2006) Can MRI rule out bladder and rectal invasion in cervical cancer to help select patients for limited EUA? Gynecol Oncol 101:244-249

15. Van Vierzen PB, Massuger LF, Ruys SH, Barentsz JO (1998) Fast dynamic contrast enhanced MR imaging of cervical carcinoma. Clin Radiol 53:183-192

16. Kinkel K, Ariche M, Tardivon AA, Spatz A, Castaigne D, Lhomme C et al (1997) Differentiation between recurrent tumour and benign conditions after treatment of gynecologic pelvic carcinoma: value of dynamic contrast-enhanced subtraction MR imaging. Radiology 204:55-63

17. Hricak H, Gatsonis C, Chi DS, Amendola MA, Brandt K, Schwartz LH et al (2005) Role of imaging in pretreatment evaluation of early invasive cervical cancer: results of the intergroup study American College of Radiology Imaging Network 6651-Gynecologic Oncology Group 183. J Clin Oncol 23:9329-9337

18. Keller TM, Michel SC, Frohlich J, Fink D, Caduff R, Marincek B et al (2004) USPIO-enhanced MRI for preoperative staging of gynecological pelvic tumours: preliminary results. Eur Radiol 14:937-944

19. Choi SH, Kim SH, Choi HJ, Park BK, Lee HJ (2004) Preoperative magnetic resonance imaging staging of uterine cervical carcinoma: results of prospective study. J Comput Assist Tomogr 28:620-627

20. Akata D, Kerimoglu U, Hazirolan T, Karcaaltincaba M, Kose F, Ozmen MN et al (2005) Efficacy of transvaginal contrastenhanced MRI in the early staging of cervical carcinoma. Eur Radiol 15:1727-1733

21. Hancke K, Heilmann V, Straka P, Kreienberg R, Kurzeder C (2008) Pretreatment staging of cervical cancer: is imaging better than palpation?: Role of CT and MRI in preoperative staging of cervical cancer: single institution results for 255 patients. Ann Surg Oncol 15:2856-2861

22. Messiou C, Morgan VA, De Silva SS, Ind TE, deSouza NM (2009) Diffusion weighted imaging of the uterus: regional ADC variation with oral contraceptive usage and comparison with cervical cancer. Acta Radiol 50:696-701

23. Charles-Edwards EM, Messiou C, Morgan VA, De Silva SS, McWhinney NA, Katesmark M et al (2008) Diffusion-weighted imaging in cervical cancer with an endovaginal technique: potential value for improving tumour detection in stage Ia and Ib1 disease. Radiology 249:541-550

24. Harry VN, Semple SI, Gilbert FJ, Parkin DE (2008) Diffusionweighted magnetic resonance imaging in the early detection of response to chemoradiation in cervical cancer. Gynecol Oncol 111:213-220 
25. Whittaker CS, Coady A, Culver L, Rustin G, Padwick M, Padhani AR (2009) Diffusion-weighted MR imaging of female pelvic tumours: a pictorial review. Radiographics 29:759-774, discussion $74-8$

26. Naganawa S, Sato C, Kumada H, Ishigaki T, Miura S, Takizawa O (2005) Apparent diffusion coefficient in cervical cancer of the uterus: comparison with the normal uterine cervix. Eur Radiol 15:71-78

27. McVeigh PZ, Syed AM, Milosevic M, Fyles A, Haider MA (2008) Diffusion-weighted MRI in cervical cancer. Eur Radiol 18:1058-1064

28. Park SO, Kim JK, Kim KA, Park BW, Kim N, Cho G et al (2009) Relative apparent diffusion coefficient: determination of reference site and validation of benefit for detecting metastatic lymph nodes in uterine cervical cancer. J Magn Reson Imaging 29:383-390

29. Xue HD, Li S, Sun F, Sun HY, Jin ZY, Yang JX et al (2008) Clinical application of body diffusion weighted MR imaging in the diagnosis and preoperative $\mathrm{N}$ staging of cervical cancer. Chin Med Sci J 23:133-137

30. Torashima M, Yamashita Y, Hatanaka Y, Takahashi M, Miyazaki K, Okamura H (1997) Invasive adenocarcinoma of the uterine cervix: MR imaging. Comput Med Imaging Graph 21:253-260

31. Liu PF, Krestin GP, Huch RA, Gohde SC, Caduff RF, Debatin JF (1998) MRI of the uterus, uterine cervix, and vagina: diagnostic performance of dynamic contrast-enhanced fast multiplanar gradient-echo imaging in comparison with fast spin-echo T2weighted pulse imaging. Eur Radiol 8:1433-1440

32. Sheu MH, Chang CY, Wang JH, Yen MS (2001) Preoperative staging of cervical carcinoma with MR imaging: a reappraisal of diagnostic accuracy and pitfalls. Eur Radiol 11:1828-1833

33. Dimopoulos JC, Kirisits C, Petric P, Georg P, Lang S, Berger D et al (2006) The Vienna applicator for combined intracavitary and interstitial brachytherapy of cervical cancer: clinical feasibility and preliminary results. Int J Radiat Oncol Biol Phys 66:83-90

34. Ferrandina G, Distefano M, Ludovisi M, Morganti A, Smaniotto D, D'Agostino G et al (2007) Lymph node involvement in locally advanced cervical cancer patients administered preoperative chemoradiation versus chemotherapy. Ann Surg Oncol 14:11291135

35. Narayan K, McKenzie AF, Hicks RJ, Fisher R, Bernshaw D, Bau S (2003) Relation between FIGO stage, primary tumour volume, and presence of lymph node metastases in cervical cancer patients referred for radiotherapy. Int J Gynecol Cancer 13:657-663

36. Hawighorst H (1999) Dynamic MR imaging in cervical carcinoma. Radiology 213:617-618

37. Scheidler J, Hricak H, Yu KK, Subak L, Segal MR (1997) Radiological evaluation of lymph node metastases in patients with cervical cancer. A meta-analysis. JAMA 278:1096-1101

38. Pandharipande PV, Choy G, del Carmen MG, Gazelle GS, Russell $\mathrm{AH}$, Lee SI (2009) MRI and PET/CT for triaging stage IB clinically operable cervical cancer to appropriate therapy: decision analysis to assess patient outcomes. AJR Am J Roentgenol 192:802-814

39. Mitchell DG, Snyder B, Coakley F, Reinhold C, Thomas G, Amendola MA et al (2009) Early invasive cervical cancer: MRI and CT predictors of lymphatic metastases in the ACRIN 6651/ GOG 183 intergroup study. Gynecol Oncol 112:95-103

40. Jimenez de la Pena M, de Vega M, Fernandez V, Recio Rodriguez M, Carrascoso Arranz J, Herraiz Hidalgo L, Alvarez Moreno E (2008) Current imaging modalities in the diagnosis of cervical cancer. Gynecol Oncol 110:S49-S54

41. Haie-Meder C, Fervers B, Chauvergne J, Fondrinier E, Lhomme C, Bataillard A et al (2000) Radiochimiothérapie concomitante dans les cancers du col de l'utérus: analyse critique des données et mise a jour des Standards, Options et Recommandations. Cancer Radiother 4:60-75

42. Gong QY, Brunt JN, Romaniuk CS, Oakley JP, Tan LT, Roberts N et al (1999) Contrast enhanced dynamic MRI of cervical carcinoma during radiotherapy: early prediction of tumour regression rate. Br J Radiol 72:1177-1184

43. Plante M, Roy M (2006) Fertility-preserving options for cervical cancer. Oncology 20:479-488, discussion 491-3 